\title{
Thoughts about the Life Work of Kenneth Arrow*
}

\author{
Péter Medvegyev
}

Kenneth Joseph Arrow is one of, and perhaps, the most important figures of modern economics. He was born on 23 August 1923 and died recently, on 21 February 2017. Perhaps it is interesting to note that although Arrow was born in the United States, his parents were Jewish immigrants from Romania, who devoted great attention to the education of their son and thought that the main tool of social progress is obtaining knowledge and holding his own in the schools.

In order to understand the intellectual legacy of Arrow and to exactly determine his place in economics, as always we must first briefly outline the precedents. Arrow's scientific achievements are based on three pillars or antecedents. The first is John von Neumann, the second is operations research, the third is the economic and political circumstances of the 1950s.

Let us start with John von Neumann. Neumann is a typical representative of the scientific generation appearing after the collapse of the Austro-Hungarian Monarchy, which fundamentally re-evaluated and transformed the world of science. Naturally, empires are not built from one day to the next nor do they disappear from one day to the next. Although the Austro-Hungarian Monarchy disappeared from the stage of history politically after World War I, it lived further for at least fifty years in schools, in scientific norms, in the world view on science and in philosophical systems. In the case of Neumann, unparalleled talent was coupled with unparalleled education. Hence, we can safely call John von Neumann the Beethoven of mathematics. His works are simultaneously characterised by elegance, universality and preciseness. Most thoughts stemming from Neumann are cited verbatim in the corresponding textbooks even after fifty years. I think that the economic works of Neumann are not among his most successful ideas, but the enormous prestige of his person opened the scientific world to the mathematical economists. Mathematical economics was born, so to speak, with the blessing and direction of Neumann. On the one hand, Neumann had raised a series of technical elements that would play a fundamental role later on, for example the

* The views expressed in this paper are those of the author(s) and do not necessarily reflect the offical view of the Magyar Nemzeti Bank.

Péter Medvegyev is a professor at the Corvinus University of Budapest. E-mail: medvegyev@uni-corvinus.hu. DOI: http://doi.org/10.25201/FER.16.2.148155 
transformation of equalities to inequality and complementarity, emphasising the duality of economic models, and the introduction of the minimax, the saddle point and economic equilibrium. On the other hand, however, and this is crucial, he accepted - and actually considered - the mathematical description and analysis of economic processes as especially important. It is often the case in mathematics that the counterexample better clarifies the statement than the statement itself. It is, therefore, worth citing the case of Louis Bachelier, who - in the course of the examination of stock exchange price movements - discovered continuous time random walk, a process and phenomenon of great importance. At the same time, the leading mathematicians in the age of Bachelier thought that stock exchange price movements are not an issue which a self-respecting mathematician has to examine, and thus the career of Bachelier cannot be called a success story, to put it mildly. Naturally, his works were re-discovered later on and became known thanks mainly to Kolmogorov. At the same time, the phenomenon discovered by him was first examined only because of its role played in physics and it became part of economic thinking much later.

The other important antecedent is the birth of the modern science of operations research. In the mathematical sense, operations research is an extension of the classic theory of optimisation. The essence of this extension is that inequalities are also allowed among the conditions, instead of just equality conditions. Naturally, the classic result, which attracted great attention at its time, was the theory and practical application of linear programming. The most important observation is the discovery of the importance of convex sets in optimisation. Previously, the necessary conditions building on differentiation had dominated in extreme value problems. Linear programming and its generalisations were built on a completely new approach. The main tool is not differentiation, but the separation theorems of convex sets and the duality theorems building on that. At that time, linear programming and its extensions were sort of an intellectual fashion, similar to mathematical finances and the pricing of derivative products forty years later. Many people thought that linear programming was a mathematical tool whose knowledge may lead to obtaining jobs and building a career easily, and thus students jostled for courses in this topic and countless textbooks on the subject were prepared, and the relevant theorems constituted the subject of discourse in the world of the academy and the corridors of universities.

Whereas the first two factors were elements appearing in the organic internal development of mathematics, the third element is a clearly political, economic and social philosophy factor. The world arising out of the bloodbath of World War I and then World War II faced a serious and historic dilemma: whether development continues in a sort of a centralised system, built on the cult of a leader, or whether a social picture building on market self-organisation will be the norm to follow. Looking back from the current vantage point, the gravity of this problem perhaps 
cannot be perceived, but this question had arisen as the fundamental problem deciding everything, which permeates everything, which determined the fate of hundreds of millions of people in the strictest sense of the word. The entire life work of Arrow can be built around answering this dilemma. According to his first result, the Arrow paradox or Arrow's impossibility theorem, it can be shown mathematically in the case of relatively evident conditions that only one method of harmonisation of the various preferences exists if the preferences of a single person, the dictator, prevail. The analysis, extension and refutation of the Arrow paradox is an independent academic field. At the same time, the extensions and mainly the refutations follow largely incorrect paths and, in my opinion, their value is very small. Arrow did not want to solve or create a mathematical problem, instead he wanted to find an explanation for one of the saddest and most exasperating phenomena of historical development. The objective of science is not the creation of a technically attractive alternative reality, but the explanation of phenomena that can be observed in the real world. With respect to society and history, the most disappointing fact is that while systems and theories attempting to redeem the life of people have been made, are made and perhaps will be made as well, all of those have made bed for the terror of autocrats and dictators. For Arrow, the notion of a dictator is not an abstract mathematical construction or a jolly logical problem. He saw dictators grinning and waving on the front page of newspapers day by day. The main experience of his young days was how the preferences of one dictator affected the people of a country of great culture, and how that remained captive even under the pressure of enormous pincers closing in hundred metres from his bunker. Not to mention the fact that the two sides of the pincers were moved by the will of another dictator. The Arrow paradox indicates the road of further progress very clearly and its message is very simple: anybody who advocates a sort of social harmony in the name of social justice opens the door for the coming of evil. A society cannot be maintained without breach of interests. Everybody who promises the opposite of this brings war, dictatorship, destruction and the Apocalypse, irrespective of his/her intentions. And it has to be emphatically emphasised that this extremely pessimistic statement is true - or is incidentally not true - not because of a trickily formulated mathematical problem. This is a historical experience which was explored, explained, illustrated and modelled by Arrow very tangibly and elegantly.

The second fundamental result of Arrow is also partly related to dictators. It is an often mentioned argument in connection with dictators that they direct societies efficiently. The classic argument is that although thousands of people were killed, imprisoned or exiled in connection with the coming to power of Napoleon III, Paris became a splendid metropolis during his rule. But we can also refer to the notable remark of the classic age that Augustus inherited a Rome built from mud and he left a Rome built from marble. And we can also recall the roads built by Hitler, or that Stalin shot a country ploughing with wooden plough into the space age. Of course, 
the legitimate question is what does efficiency mean? Everything has its price, and cost and benefit must be considered simultaneously in the case of efficiency. It is thus evident that we should identify the efficient statuses with Pareto efficiency, i.e. with such statuses in which the result cannot be increased further with the given level of costs, or in the case of which costs cannot be decreased further in the case of maintaining the results. According to the fundamental theorem of welfare economics, stemming from Arrow and bearing his name, equilibrium statuses are efficient, and in the case of certain conditions efficient statuses can be transformed to equilibrium status. It should be emphasised that in the Arrow theorem we can choose from the individual efficient statuses with the income distribution parameters. The key idea is the notion of the equilibrium, which is the intellectual counterweight of the "ideal" status prescribed and planned by the dictator. The notion and widespread use of equilibrium is questioned by many in economic theory, perhaps not even completely without foundation. Thus, it is worth talking about it a bit. First, it is worth noting that the notion of the equilibrium is used in several different senses. In most cases, it is customary to think of equilibrium in a dynamic sense, associating as example to the balls rolling down from a slope. However, the concept of equilibrium used by Arrow is not the result of a dynamic movement, and the equilibrium status does not represent an ideal status by any means. In fact, it is more like a trap, from which no escape is possible without external assistance. When dealing with social issues the first obvious question is why the losers of the social system come to terms with their situation, and why they do not attempt to do something for example against their poverty and unfavourable situation? The answer is very simple: because they are in an equilibrium situation, and thus they cannot escape from this status on their own, since their present status is the best status achievable for them, provided that their environment does not change their situation either. No matter that someone is unemployed and no matter that there are work opportunities at another place, moving from one place to another place is impossible because of high real estate prices. In the Arrow model everybody optimises, but they obviously do this with the specific distribution of wealth and incomes. People are not happy in the equilibrium, it is only that they cannot change their situation. Arrow's fundamental theorem of welfare economics can be considered as a key theorem in two senses. On the one hand, the mathematical tools and conditions necessary for the verification of the existence of the equilibrium, his later main work, appear here; on the other hand, this result records the sphere of possible social movements, since it connects the notions of the equilibrium and efficient statuses. It is worth indicating in terms of mathematics that it is again a very simple theorem. Indeed, the proof includes the apparent application of the theorem about the separation of convex sets. The researchers of the age routinely applied such types of considerations in the literature of linear programming. What makes the theorem important is the fundamental description of the operation of the society and not the mathematical contents or the exact 
discussion of the conditions. On the one hand, efficiency is perceived not only in dictatorial systems, market competition also results in the efficient distribution of resources, but on the other hand, it sheds light on another important factor, namely that not only the individual is responsible for the destiny of the individual. Poverty and distress are the responsibility of the entire society, since the destitute have no other choice in an equilibrium. Moreover, which of the efficient statutes is realised essentially depends on the other parameters of the society, mainly on the distribution of wealth and incomes. The fundamental theorem of welfare economics is not simply a mathematical model, but a framework for thinking, in which the social problems can be formulated and discussed. If the set of efficient and equilibrium statuses is identical, it is sufficient to leave the market statuses on their own and concentrate on the indirect handling of environmental conditions in the course of state intervention. The separation of economic participants aiming for equilibrium and economic policy influencing the exact parameters of the equilibrium is the main starting point of modern economic thinking. But the opposite reasoning is possible, too. If economic policy influences prices and market relations not indirectly, it inevitably results in an inefficient status. In other words, fixing prices restricts equilibrium mechanisms and entails only negative consequences, and these methods do not assist the destitute, whose situation we would like to improve in the given case.

With this, we have reached the main result of his life's work, the proof of existence of economic equilibrium. According to a legend known at all the universities of the world, a professor not much liked by the students discussed the fascinating properties of a mathematical structure in hundreds of papers, until eventually a first-year student of an end-of-the-world university proved that the structure is either empty or it only includes some trivial elements. Hence, the mathematical proof of the existence of economic (general) equilibrium had been an old wish of economic thinking. The problem had been stated in a more or less exact mathematical language by French economist Léon Walras as early as the 1870s. The life work of Walras caught on in the English-speaking countries relatively slowly, but in the 1950s the model and the related mathematical problem was already known by everyone. Naturally, it was not Arrow and his co-author Gerard Debreu who were the first to prove the existence of equilibrium in a mathematical model motivated in terms of economics. The line of predecessors goes back to John von Neumann, who introduced the notion of the saddle point in the framework of game theory, which was later generalised by Nash to the notion of the Nash equilibrium, bearing his name today. Actually, Nash's theorem, which verifies the existence of the Nash equilibrium, already includes in an extremely abstract form the conditions providing the existence of the equilibrium of the model of Arrow and Debreu. Building on the article of Nash, Arrow and Debreu described a schematic economic model and showed that in the model described by them the existence of 
the equilibrium defined by them follows from the existence of the Nash equilibrium of the Nash abstract game theory model. The mathematical key of the solution is the Brouwer fixed-point theorem and the generalisation of that. The most famous generalisation of the theorem is known as the Kakutani fixed-point theorem. It is worth emphasising that Kakutani proved his theorem to simplify the proof of the Neumann fixed-point theorem. On the other hand, Neumann verified the existence theorem of the growth model bearing his name with his fixed-point theorem. The Arrow-Debreu model divides economic participants into two groups. On the one hand, there are consumers, who maximise the utility that can be achieved by them given their income. In other words, they solve an extreme value problem with parametric conditions. The most important parameter of the problem is income, which stems partly from selling their wealth, and partly from the part they receive from the profit produced by the producers, the other group of the participants. Producers and consumers concurrently create demand and supply for the products appearing in the model. It is customary to refer to this model as the model of general equilibrium since equilibrium exists concurrently on all the markets, i.e. generally. The equilibrium factor which settles the two sides of demand and supply is the movement of prices. In other words, according to the model, only prices are capable of settling demand and supply and create the market equilibrium.

It is not an exaggeration to state that the proof of the existence of the general equilibrium was the most significant achievement in economic theory in the 1950s. The fact and method of the proof provided extreme self-confidence to contemporary economic researchers. After eighty years, a mathematical problem motivated economically was proven elegantly and clearly. At the same time, it also turned out that the pioneering contribution of leading mathematicians was necessary for the solution. Not without foundation, mathematics has always been the sample science for scientific researchers, with a glorious pedigree of thousands of years with its axiomatic method. There is some fuzzy element if something cannot be captured mathematically. Naturally, there are important areas of knowledge and there are the sciences. And although economics had always been a storehouse of useful knowledge (who would dare deny this), with the proof of the Arrow-Debreu model, it entered the Valhalla of scientific theories, or at least many people thought so.

For my part I, prefer to concentrate on the shortcomings of the model and analyse with what conditions the verification of the existence of the equilibrium was successful. The most important conditions are the various convexity conditions. All of the sets and functions appearing in the model are in some way convex or concave. According to the interpretation of the convexity conditions, this means that there are decreasing returns to scale. In addition to decreasing returns to scale, the other deficiency of the model is that the representation of time and randomness is extremely schematic in the model. Despite all of these, not much success has been 
achieved - during the sixty years passed since then - in modifying the conditions of the model, and in exceeding that either mathematically or economically. Tens of thousands of dynamic and stochastic generalisations have been born, but the basic construction can be perceived in all the generalisations in a way that cannot be surpassed. Everyone who was alive and active received a Nobel prize in economics for the development of the Arrow-Debreu model and the precedent of that, and, without doubt, it provides an example for every economist until today with respect to how an economic problem has to be stated and solved mathematically.

After the verification of the existence of the Walras economic (general) equilibrium, investigation of the uniqueness and stability of the equilibrium has arisen evidently. However, the examinations in this direction have essentially produced only negative results. The reason for this is that the so-called Walras Law, which plays a key role in the Arrow-Debreu model, does not sufficiently restrict the dynamic properties of market systems. According to the statement of the Walras Law, in the case of each price system, irrespective of whether the price system is an equilibrium price system or not, the amount of value of demand and supply is always identical, since each economic participant represents such demand which is identical to its income, and income is generated in such a way that the economic participants offer some resource. In the language of mathematics, this means that the excess demand function, defined as the difference of demand and supply, is always perpendicular to the price system belonging to it. In terms of mathematics, the problem stems from the fact that it can be shown that each function that has the property that the argument of the function is perpendicular to the value of the function is an excess demand function of a suitable Arrow-Debreu model. But this is only one of the countless mathematical considerations that have been inspired by the model. It is difficult to find a result of modern economics that is not directly related to the Arrow-Debreu model. In addition to the direct connections, the style and approach followed by the authors decisively modified the science of economics.

Naturally, the life work of Arrow was not restricted to the development of the general equilibrium theory, but it is indisputable that this is his most famous work and these results are cornerstones of thinking about the economy that have not been worn by the extensive examination of the last decades which has been steadfast and extremely detailed. Kenneth Arrow was undoubtedly an unparalleled thinker, whose greatness in the history of science can only be measured with John von Neumann, his great predecessor. I think that this comparison is an honour with respect to both giants of science. Similarly to Neumann, Arrow has left his mark on countless areas. His wide-ranging interest and exceptional mathematical talent made it possible to create enduring results not only in economics, but in the area of pure mathematics as well. I will not undertake to enumerate his achievements, as the number of these is too great and too wide-ranging for me. But I think that 
I can undertake to outline and evaluate the method of approach followed by him and demonstrated as an example. It is a scientific principle of the methodology permeating the works of Arrow that he thought that economics was mature enough to state its questions in the form of mathematical models. Moreover, he examined the mathematical theories belonging to the models with great joy and devotion. He had the unrivalled ability that he could see the mathematics in economics problems and the economics in mathematics. Mathematical talent is a common ability that can be developed in most people with good upbringing and appropriate education. The main problem of mathematics is that it is essential for its development that it should not separate in some way from the external impulses and it should be motivated sufficiently by other sciences. The generation of economists whose best known and most important figure was Arrow has had an enormous effect on the development of mathematics, since it opened the sphere of applications of mathematics and broke the connection of mathematics and the natural sciences in a good sense. In terms of modern mathematics, economics is a legitimate area of application to exactly the same extent as physics or any other natural scientific problem. Moreover, several natural scientists observed that the mathematical exactness of modern economists and their commitment to mathematical precision is much greater than that of their natural scientific colleagues. I also often hear the remark that students with an economics degree are not behind students majoring in other areas in terms of their preparedness in mathematics, computer science and statistics. If this is true, one of the reasons for this is the model which was received by the society of economists from the predecessors. From those predecessors who had learnt it mostly from Kenneth Arrow how a problem of economics has to be stated and analysed. At the same time, let me make a last remark. Style is often more important than the result itself. Modern economics is undoubtedly written in the language of mathematics. Yet language often restricts the message. In the case of Arrow, the classic philosophical notions of content and form were balanced and neither side repressed the other. The key of his greatness lies exactly in this equilibrium. 